\title{
Iranian Environmental Health Strategies and the Challenges Against COVID-19
}

\author{
Tooraj Massahi (iD) ${ }^{1,}$, Mohammad Jasimi (iD) ${ }^{1}$, Negar Faridi (iD) ${ }^{1}$ and Sheida Atashkar (iD ${ }^{2}$ \\ ${ }^{1}$ Student Research Committee, School of Public Health, Kermanshah University of Medical Sciences, Kermanshah, Iran \\ ${ }^{2}$ School of Public Health and Safety, Shahid Beheshti University of Medical Science, Tehran, Iran \\ "Corresponding author: Student Research Committee, School of Public Health, Kermanshah University of Medical Sciences, Kermanshah, Iran. Email: \\ toorajmassahi@gmail.com
}

Received 2021 April 02; Revised 2021 April 24; Accepted 2021 May 05.

Keywords: Environmental Health, COVID-19, Pandemic, Coronavirus, Iran

\section{Introduction}

In December 2019, a new infectious respiratory disease was reported in Wuhan city, located in Hubei province (China). On December 31 of the same year, the World Health Organization (WHO) Headquarters officially reported the disease, and on February 11, 2020, the WHO named the infectious disease COVID-19 (Coronavirus disease 2019). COVID-19 was announced a pandemic by the WHO on March 11, 2020. COVID-19 is caused by a newly discovered class of coronaviruses known as SARS-CoV-2 (severe acute respiratory syndrome coronavirus 2) (1-4). The new COVID19 is highly contagious and has rapidly spread globally. It is the cause of an acute respiratory disease, which has infected and killed thousands of people around the world (5), posing a fragile challenge to the resilience of the health system in every country.

The world is currently facing a unique health crisis (6). According to the Worldometer website published statistics on March 15, 2020 (7), there has been a minimum of 6,572 total deaths and 214,738 total confirmed cases of COVID-19 worldwide. On March 15, 2021, the confirmed cases of COVID-19 reached 120,832,193 with a mortality rate of 2,673,254; these numbers are increasing alarmingly.

Health is a key element for the development and progress of every society. In a pandemic, comprehensive information must be attained about the risk factors and potential solutions within the community (6). In Iran, the assessment of health challenges (especially environmental health) during the COVID-19 pandemic could be a lesson for future generations and provide health authorities with solutions to improve COVID-19 control policies.

\section{Arguments}

On March 15, 2021, Iran reported a total of 1,754,933 and daily 7,980 confirmed cases of COVID-19, including a total of 61,330 and daily 100 COVID-19 deaths (7). Therefore, it is essential to prioritize health over treatment (especially environmental health) as a cost-efficient, comprehensive, and accessible principle. The International Federation of Environmental Health (8) has also introduced the slogan of the World Environmental Health Day 2020 as "Environmental health, the key strategy of the health system in combating the spread of diseases."

In Iran, environmental health professionals are key to implementing preventive measures against COVID-19 and play a pivotal role in controlling the disease since the onset. As a result, the presence of environmental health professionals in the main group of anti-Corona headquarters decision-makers could be highly effective.

Environmental health challenges are a pressing issue, which could adversely affect the severity and duration of the COVID-19 pandemic. As the health community is exposed to a highly contagious virus, hygienic practices are considered the simplest and most effective approach to the prevention of COVID-19. Early in the pandemic, the disorganization of health scientists to develop practical and operational guidance led to the development of various guidelines on similar topics. Due to extensive and unmanaged activities, part of the time and resources allocated to this purpose was wasted. Furthermore, unprofessional measures such as the unnecessary disinfection of cities, roads, and villages caused the waste of resources and their use in a low-priority manner in Iran.

Attention to research and scientific committees is a cost-effective strategy to increase preparedness in crisis 
management when the community faces budget shortages, poor infrastructures, and a lack of public knowledge. The lack of virology research laboratories in several countries (including Iran) has also led to inadequate field research. Moreover, the insufficient knowledge of coronavirus transmission and the fear of contamination due to contact with COVID-19 corpses led to hasty and inaccurate instructions and directed the corpses to be buried in unsuitable conditions with lime to the depth of five meters. The concern was such that even relatives of the deceased refused to approach the bodies. Under such circumstances, researchers investigated the contaminated bodies and determined their contamination level with COVID-19, which resulted in the development of guidelines that corrected the mismanagement of the burial of the patients who died due to COVID-19.

In the urban transport fleet, there are high-contact and important points that may provide the conditions for the transmission of the coronavirus from an infected person. Two groups of people are extremely vulnerable in the current pandemic; the first group is the passengers who do not observe a distance of at least one meter from others in crowded areas and do not often use appropriate personal protective equipment (PPE). The second group is the drivers of urban transportation systems, many of whom have died due to contracting the coronavirus infection on their own or from their families. Therefore, appropriate health protocols were implemented for the use of PPE such as face masks and gloves by drivers and passengers.

In terms of providing personal protective and safety equipment, disinfectants, practical and comprehensive protocols, and experienced manpower, medical centers are faced with inadequate infrastructures to become specialized for coronavirus care. The preparedness of the health structures, facilities, and equipment and the preplanned training and education of the public, medical experts, and hospital staff could reduce interference with the executive planning in this regard, as well as the rapid spread of the coronavirus. The hospitals and healthcare centers that were entirely dedicated to COVID-19 patient care during the current pandemic had not been designed for COVID-19 care. As a result, their ventilation systems were not of negative pressure. The infectious agent has potential airborne transmission, and the air conditioning systems of numerous hospitals could cause the spread of the virus in different wards. Therefore, attention must be paid to the design and implementation of proper air conditioning and natural ventilation systems in hospitals.

The increased production of medical waste and frequent consumption of plastics in hospitals and healthcare centers have also caused various issues in the conventional solid waste management systems, which are designed for stable and normal conditions. The release of gloves and face masks used in cities, streets, and public places has shown that if countries remain unaware of the debilitative effects of the current pandemic on the environment, new secondary problems may arise, the control of which will be challenging and time-consuming. Shortly after the current pandemic began, research projects and scientific committees greatly helped take effective measures in this regard.

\section{Conclusions}

Despite the growth and development in medical and health technologies prior to and during the COVID-19 pandemic, special attention should be paid to primary healthcare with an emphasis on environmental health. Evidently, precautionary measures such as using face masks in public areas and public closed spaces, washing / disinfecting hands after touching surfaces, and observing social distance have gained proper attention.

To effectively manage COVID-19, reduce the casualties, and prepare for the pandemics that may occur in the future, health and government leaders must adopt proper preventive policies and take practical steps toward providing and developing effective environmental health infrastructures. Moreover, public participation in the implementation of preventive programs should be encouraged in an informed, committed manner. In order to achieve these goals, we should address the hygiene and health education issues that affect community health and strengthen the framework of the national healthcare system, while using the perspectives of environmental health professionals and their guidance on the preventive measures in this regard. Disseminating the belief that "prevention is better than cure". Could also have a tremendous impact on the resolution of health crises such as COVID-19.

\section{Footnotes}

Authors' Contribution: All authors have been active in providing the reported paper. T.M. wrote the manuscript with support from M.J., N.F., and Sh.A. All authors discussed the results and contributed to the final manuscript.

Conflict of Interests: There is no conflict of interest. Funding/Support: There is no funding/support.

\section{References}

1. Awasthi A, Avasthi S. Outbreak of Novel Corona Virus in India: Lethal Pandemic. Acta Sci Agric. 2020;4(5):44-5. doi: 10.31080/asag.2020.04.0839. 
2. Tripathi A, Tyagi VK, Vivekanand V, Bose P, Suthar S. Challenges, opportunities and progress in solid waste management during COVID19 pandemic. Case Studies in Chemical and Environmental Engineering. 2020;2:100060. doi:10.1016/j.cscee.2020.100060.

3. Chan JF, Yuan S, Kok KH, To KK, Chu H, Yang J, et al. A familial cluster of pneumonia associated with the 2019 novel coronavirus indicating person-to-person transmission: A study of a family cluster. Lancet. 2020;395(10223):514-23. doi:10.1016/S0140-6736(20)30154-9. [PubMed: 31986261]. [PubMed Central: PMC7159286].

4. Farnoosh G, Alishiri G, Zijoud SH, Dorostkar R, Farahani AJ. [Understanding the severe acute respiratory syndrome Coronavirus 2 (SARS-CoV-2) and Coronavirus disease (COVID-19) based on available evidence-a narrative review]. Mil Med. 2020;22(1):1-11. Persian.

5. Verma A, Prakash S. Impact of COVID-19 on environment and society.
Journal of Global Biosciences. 2020;9(5):7352-63.

6. Diaz de Leon-Martinez L, de la Sierra-de la Vega L, Palacios-Ramirez A, Rodriguez-Aguilar M, Flores-Ramirez R. Critical review of social, environmental and health risk factors in the Mexican indigenous population and their capacity to respond to the COVID-19. Sci Total Environ. 2020;733:139357. doi: 10.1016/j.scitotenv.2020.139357. [PubMed: 32416536]. [PubMed Central: PMC7215151].

7. Worldometers. COVID-19 Coronavirus pandemic. USA: Worldometers; 2021, [cited 2021]. Available from: https://www.worldometers.info/ coronavirus/.

8. International Federation of Environmental Health. International Federation of Environmental Health. London, UK: International Federation of Environmental Health; 2021, [cited 2021]. Available from: https: //www.ifeh.org/. 